ARTICLE

Received 13 Jun 2016 | Accepted 22 Dec 2016 | Published 15 Feb $2017 \quad$ DOl: 10.1038/ncomms14402 OPEN

\title{
Hyperspectral infrared nanoimaging of organic samples based on Fourier transform infrared nanospectroscopy
}

Iban Amenabar ${ }^{1}$, Simon Poly ${ }^{1,2}$, Monika Goikoetxea1,3, Wiwat Nuansing ${ }^{1}$, Peter Lasch $^{4}$ \& Rainer Hillenbrand ${ }^{5,6}$

Infrared nanospectroscopy enables novel possibilities for chemical and structural analysis of nanocomposites, biomaterials or optoelectronic devices. Here we introduce hyperspectral infrared nanoimaging based on Fourier transform infrared nanospectroscopy with a tunable bandwidth-limited laser continuum. We describe the technical implementations and present hyperspectral infrared near-field images of about 5,000 pixel, each one covering the spectral range from 1,000 to $1,900 \mathrm{~cm}^{-1}$. To verify the technique and to demonstrate its application potential, we imaged a three-component polymer blend and a melanin granule in a human hair cross-section, and demonstrate that multivariate data analysis can be applied for extracting spatially resolved chemical information. Particularly, we demonstrate that distribution and chemical interaction between the polymer components can be mapped with a spatial resolution of about $30 \mathrm{~nm}$. We foresee wide application potential of hyperspectral infrared nanoimaging for valuable chemical materials characterization and quality control in various fields ranging from materials sciences to biomedicine.

\footnotetext{
${ }^{1}$ CIC nanoGUNE, 20018 Donostia-San Sebastián, Spain. ${ }^{2}$ IFIB - Interfaculty Institute of Biochemistry, 72076 Tübingen, Germany. ${ }^{3}$ Metallic Surfaces Unit, IK4-CIDETEC, 20014 Donostia-San Sebastián, Spain. ${ }^{4}$ Department of Proteomics and Spectroscopy (ZBS6), Robert Koch Institut, 13353 Berlin, Germany. ${ }^{5} \mathrm{CIC}$ NanoGUNE and UPV/EHU, 20018 Donostia-San Sebastián, Spain. ${ }^{6}$ IKERBASQUE, Basque Foundation for Science, 48013 Bilbao, Spain. Correspondence and requests for materials should be addressed to R.H. (email: r.hillenbrand@nanogune.eu).
} 
nfrared (IR) vibrational spectroscopy ${ }^{1}$ is a valuable tool for materials characterization in widely different fields, ranging from polymer sciences to biomedical imaging ${ }^{2-5}$. It allows for highly sensitive studies of chemical and structural properties, among others. Diffraction, however, limits its spatial resolution and sensitivity, thus preventing the study of nanoscale materials and composites, as well as of single biological macromolecules. The IR diffraction limit can be circumvented, among other techniques ${ }^{6-8}$ by infrared scatteringtype scanning near-field optical microscopy (IR s-SNOM) 9,10 and its extension to nanoscale Fourier transform infrared (nano-FTIR) spectroscopy ${ }^{1-19}$, which enable infrared imaging and spectroscopy with nanoscale spatial resolution, respectively.

s-SNOM and nano-FTIR spectroscopy are based on atomic force microscopy (AFM), where either monochromatic or broadband infrared radiation, respectively, is scattered by a metallic AFM tip. The tip acts as an antenna and concentrates the incident infrared field at the very tip apex to a nanoscale spot size (nanofocus) ${ }^{10,12,20}$, locally exciting infrared-vibrational resonances in the sample that modify the tip's scattered field. In s-SNOM, the tip-scattered field is recorded with an interferometer as a function of tip position, yielding two-dimensional (2D) monochromatic infrared amplitude and phase images. The spatial resolution is determined by the radius of the tip apex, which is typically in the range of $10-30 \mathrm{~nm}$. In nano-FTIR spectroscopy, the tip is illuminated with the radiation from a thermal source ${ }^{12,20,21}$, an infrared laser continuum ${ }^{11,14,22-25}$ or a synchrotron ${ }^{17-19,26}$. Fourier transform spectroscopy of the scattered light yields a local infraredvibrational spectrum (point spectrum) of the sample surface with a spatial resolution similar to s-SNOM. Because of an asymmetric spectrometer design (that is, tip and sample are located in one of the interferometer arms $\left.{ }^{14,16,23}\right)$, both amplitude and phase (respectively real and imaginary part) of the scattered field are measured. It has been shown that the phase, respectively imaginary part of nano-FTIR spectra of organic materials can be well interpreted with the help of far-field FTIR absorbance spectra, allowing for materials characterization and identification based on standard infrared references ${ }^{14,15,18,27}$.

Spatially resolved spectroscopic information can be obtained by sequential s-SNOM imaging of one and the same sample area at different infrared frequencies using a tunable monochromatic laser. From the stack of images, an infrared spectrum can be constructed at each pixel ${ }^{28-34}$. Typically, tunable monochromatic $\mathrm{CO}_{2}$ or $\mathrm{cw}$-quantum cascade lasers are used as infrared source. As they can be tuned only over a relatively small spectral width of about $200 \mathrm{~cm}^{-1}$, several lasers are required to cover the whole mid-infrared spectral range. On the other hand, frequencies below $900 \mathrm{~cm}^{-1}$ are barely reached with table-top cw-lasers. Further, the nonlinear and typically uncontrolled drift between sample scanner and tip, as well as the tip wear due to the numerously repeated sample scanning, is challenging the construction of accurate local broadband infrared spectra.

Spectroscopic infrared nanoimaging, in principle, can be also achieved by recording a nano-FTIR spectrum at each pixel of a 2D image. Advantages would include the availability of continuous broadband radiation also below $900 \mathrm{~cm}^{-1}$ (provided by thermal sources ${ }^{12,21}$, synchrotrons ${ }^{18,26,35}$ and infrared laser continua $\left.{ }^{14,15,22,23,36,37}\right)$, and that repetitive scanning and related problems (tip wear) could be avoided. However, the low-spectral irradiance of many broadband infrared sources is strongly challenging nano-FTIR based hyperspectral infrared nanoimaging, that is, the recording of a large $2 \mathrm{D}$ array of nano-FTIR spectra. For example, synchrotrons provide ultra-broadband infrared radiation with a spectral irradiance of about $0.2 \mathrm{~W} \mathrm{~cm}^{-2} \mathrm{~cm}^{-1}$ (ref. 18) at mid-infrared frequencies, which is several orders of magnitude weaker than that of mid-IR quantum cascade lasers used for s-SNOM imaging. The reported time for recording nano-FTIR spectra of materials with a strong infrared response (for example, phonons in $\mathrm{SiO}_{2}$ ) amounts to about $1 \mathrm{~min}$ (ref. 18), which is significantly increased for weakly absorbing organic substances. The recoding of a hyperspectral image of about $100 \times 100=10,000$ pixel would require many tens of hours, thus challenging the mechanical and optical stability of nano-FTIR setups. Alternatively to synchrotrons, mid-infrared laser continua based on difference frequency generation (DFG) can be applied for nano-FTIR spectroscopy $14,15,22,23,36,37$. These table-top lasers provide tunable infrared radiation of several $100 \mathrm{~cm}^{-1}$ bandwidth and a spectral irradiance that can be much higher compared with synchrotrons. However, hyperspectral infrared nanoimaging with such lasers has not been achieved yet. Reasons include the non-negligible sample drift during spectra acquisition, as well as the still relatively small spectral bandwidth that requires the combining of multiple bandwidth-limited nano-FTIR spectra at each pixel.

Here we introduce and describe hyperspectral infrared nanoimaging by nano-FTIR spectroscopy with a tunable laser continuum. It is based on recording and stitching together multiple bandwidth-limited nano-FTIR spectra at each pixel of a $2 \mathrm{D}$ sample area, which is enabled by sample drift correction during data acquisition. Specifically, we use a tunable DFG laser continuum $\left(350 \mathrm{~cm}^{-1}\right.$ effective bandwidth) to record nanoscale-resolved hyperspectral infrared images comprising about 5,000 nano-FTIR spectra that cover the spectral range from 1,000 to $1,900 \mathrm{~cm}^{-1}$. We verify the technique with a threecomponent polymer blend and a hair cross-section, and demonstrate that standard multivariate analysis of the hyperspectral images can be performed. As a first application, we demonstrate the much sought-after possibility of mapping chemical interaction between polymer components with nanoscale spatial resolution.

\section{Results}

Set-up and methodology for hyperspectral infrared nanoimaging. We developed hyperspectral infrared nanoimaging (Fig. 1) using a commercial nano-FTIR set-up (Neaspec GmbH). It is based on an AFM, where a standard Au-coated tip is vertically vibrating at the mechanical resonance frequency $\Omega$ of the cantilever. The tip is illuminated with a DFG-generated mid-infrared laser continuum of about $350 \mathrm{~cm}^{-1}$ spectral bandwidth, which centre frequency is tuneable between 1,200 and $1,600 \mathrm{~cm}^{-1}$ (see Fig. 1a and Supplementary Note 1). The tip-scattered light is recorded with a Michelson interferometer. To perform background-free nano-FTIR spectroscopy ${ }^{14,38,39}$, the detector signal is demodulated at a higher harmonic $n$ of the tip's oscillation frequency, $n \Omega$, and recorded as a function of the reference mirror position $d$, yielding the interferogram $I(d)$. Throughout this work, the demodulation order was $n=3$. Because tip and sample are located in one interferometer arm, Fourier transform of $I(d)$ yields amplitude $s_{s}(\omega)$ and phase $\varphi_{\mathrm{s}}(\omega)$ spectra ${ }^{14,27}$. To obtain normalized nano-FTIR spectra $s=s_{\mathrm{s}} / s_{\text {ref }}$ and $\varphi=\varphi_{\mathrm{s}}-\varphi_{\text {ref }}$, the tip is positioned on a reference area on the sample (typically a clean gold or silicon surface $)^{14,23}$ to record the reference spectra $s_{\text {ref }}(\omega)$ and $\varphi_{\text {ref }}(\omega)$.

For hyperspectral nanoimaging we record interferograms at each pixel $(x, y)$ of a $2 \mathrm{D}$ area of the sample surface (Fig. 1b). Subsequent Fourier transform and normalization to reference 
a

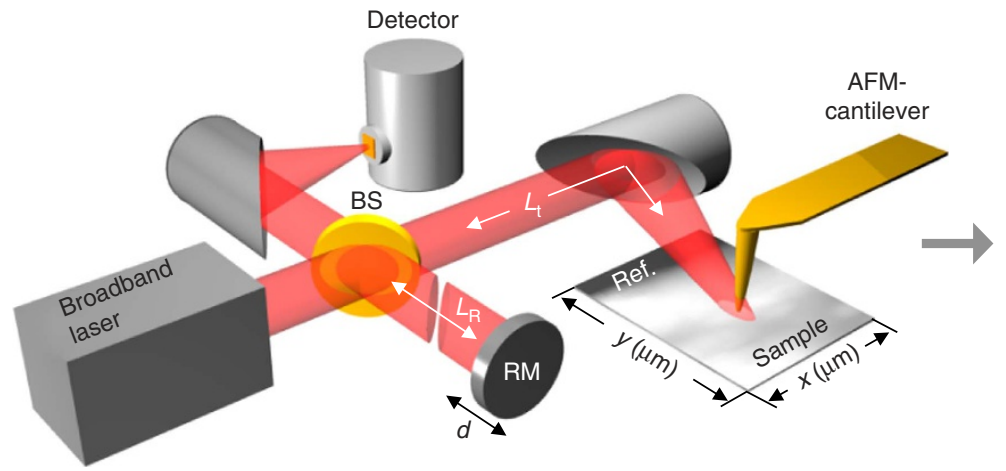

b

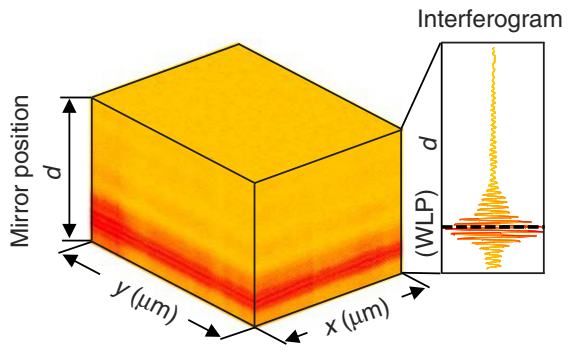

C

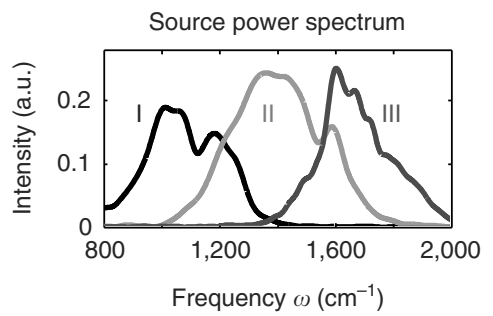

Frequency $\omega\left(\mathrm{cm}^{-1}\right)$

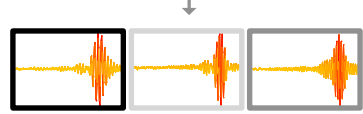

$\downarrow$

Local sample spectrum

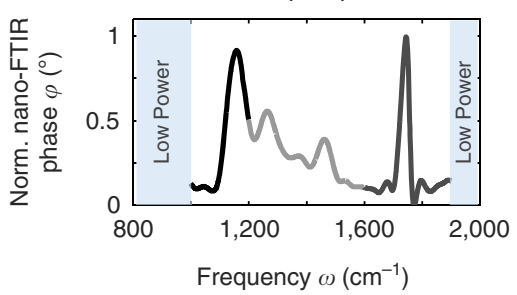

d
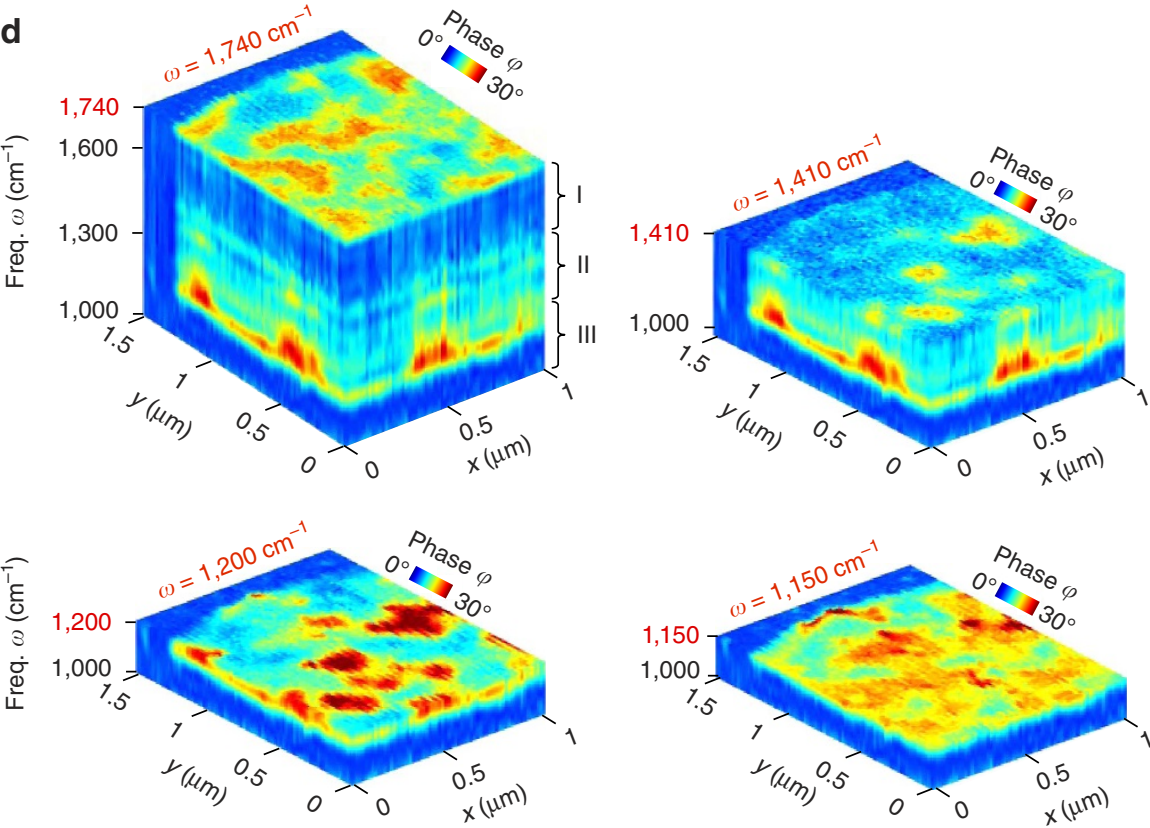

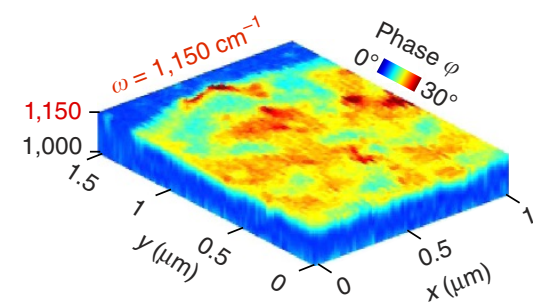

Figure 1 | Hyperspectral infrared nanoimaging. (a) Set-up employing a mid-infrared laser continuum source for tip illumination. The tip scans the sample surface while at each pixel the light backscattered from the tip is analysed with a Michelson interferometer that is operated as a Fourier transform spectrometer. The setup comprises a beam splitter (BS, uncoated ZnSe), a reference mirror (RM) and a detector. (b) 2D array of interferograms. (c) Top: Output spectra of the source. Middle: Interferograms recorded for each output spectrum. Bottom: Broadband nano-FTIR spectrum composed by three nano-FTIR spectra obtained by Fourier transform of the corresponding interferograms and normalization to a reference spectrum (middle panel). (d) Hyperspectral infrared data cubes of spectral resolution of $35 \mathrm{~cm}^{-1}$, cut at different frequencies $\omega$. They show the normalized phase $\varphi$ of the tip-scattered light as a function of position $(x, y)$ and frequency $\omega$.

spectra yields a $2 \mathrm{D}$ array of nano-FTIR spectra with a spectral bandwidth determined by the output spectrum of the DFG laser source, that is, a hyperspectral data cube $A(x, y, \omega)$ where $x$ and $y$ represent two spatial dimensions and $\omega$ the frequency (spectral dimension). For the present work, we recorded and studied nano-FTIR phase spectra $\varphi(\omega)$, as they are related to the sample's infrared absorption ${ }^{16,27}$, the later being typically analysed when infrared spectroscopy of organic materials is performed. To increase the spectral bandwidth, we record data cubes $A^{\mathrm{k}}=\varphi^{\mathrm{k}}(x, y, \omega)$ at three different DFG output spectra (indicated by the index $\mathrm{k}=\mathrm{I}, \mathrm{II}, \mathrm{III}$ and shown in the upper panel of Fig. 1c) and stitch together at each pixel the corresponding normalized nano-FTIR phase spectra (Fig. 1c, lower panel). As a result, a hyperspectral data cube $A$ is obtained. In Fig. $1 \mathrm{~d}$ we show the hyperspectral infrared data cube of a three-component polymer blend on a silicon substrate, exhibiting a spatial resolution of about $30 \mathrm{~nm}$ (further details see below). Cutting the cube at different infrared frequencies $\omega$ yields monochromatic infrared images. They clearly reveal a rich variety of spectrally and spatially varying features, indicating that neither individual point spectra nor individual monochromatic images provide the full information content contained in the hyperspectral data of this sample.

In the following we describe the key implementations of our technique (for more technical details see Supplementary Notes 1-6).

We increased the data acquisition speed by improving the signal-to-noise ratio (SNR) of the individual nano-FTIR spectra. To that end, the output power of our mid-IR laser continuum (previously reported in refs 14,23) was increased from about $100-250 \mu \mathrm{W}$ to $600 \mu \mathrm{W}$. Further, because of the asymmetric interferometer set-up (that is, the sample is located in one of the interferometer arms), we record only one half of the interferogram (Fig. 1b), as the other one does not contain spectroscopic information about the sample (see Supplementary Note 2 and Supplementary Fig. 1). With our improved laser source we 


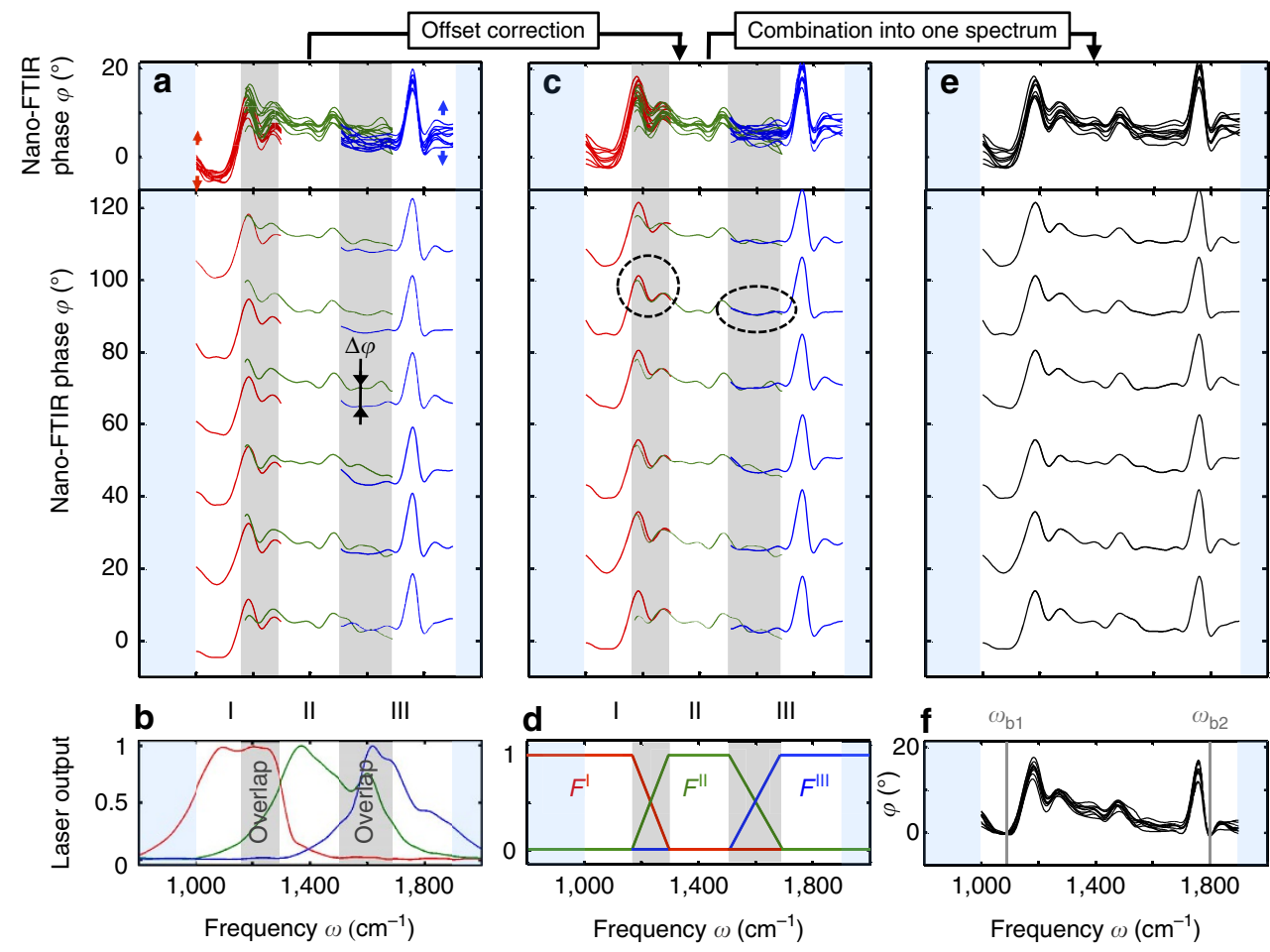

Figure 2 | Stitching of bandwidth-limited normalized nano-FTIR spectra. (a) Bandwidth-limited normalized nano-FTIR phase spectra from six subsequent pixels of the hyperspectral data cube shown in Fig. 1. (b) Laser output spectra. (c) Spectra of a after offset correction. (d) Functions $F^{\prime}$, $F^{\prime \prime}$ and $F^{\prime \prime \prime}$.

(e) Broadband nano-FTIR spectra after stitching. (f) Broadband nano-FTIR spectra after stitching and baseline correction. The top panels of a,c,e show all normalized spectra plotted on top of each other, while the bottom panels show the same spectra plotted for each pixel separately.

succeeded to obtain about $350 \mathrm{~cm}^{-1}$ broad nano-FTIR spectra of organic materials in $1.66 \mathrm{~s}$, which is more than one order of magnitude faster than what has been previously achieved with DFG and synchrotron radiation ${ }^{14,18,23}$.

For accurate and reliable normalization of the nano-FTIR spectra, the reference spectra need to be recorded under the same experimental conditions. Due to slight spectral fluctuations of the laser continuum and drift of the interferometer arms (see discussion below, Supplementary Note 5 and Supplementary Fig. 3), however, the conditions may vary between sample and reference measurements. For that reason, we regularly acquire interferograms of a reference area while recording the data cube. This can be achieved, for example, by recording the data cube such that each line contains a clean reference area (for example, silicon, marked Ref. in Fig. 1a where the line scans are parallel to the $y$-axis). The nano-FTIR spectra of each line are then normalized to the reference spectrum included in this line.

Depending on the number of individual spectra, the acquisition of a data cube $A^{\mathrm{k}}$ may still take several minutes to few hours. To avoid artifacts due to sample drift, we adapted concepts known from other imaging techniques (for example, electron energy loss spectroscopy mapping or $\mathrm{AFM}^{40}$ ). In brief, after $n$ spectroscopic line scans (that is, recoding nano-FTIR spectra along $n$ complete lines of the image), the sample is repositioned, yielding a data cube in which sample drift has been compensated (see Supplementary Note 3 and Supplementary Fig. 2). To this end, the spectroscopic data acquisition is stopped after each block of $m$ line scans and a topography image of the sample including a reference point is recorded. The position of the reference point relative to its previous position is measured. The sample scanner is accordingly repositioned. We chose the number $m$ such that the sample drift (determined essentially by the temperature stability of the setup) during the $m$ line scans is smaller than the spatial resolution of about $30 \mathrm{~nm}$. In the experiments presented in Figs 3 and 4 we set $m=2$ and $m=4$, respectively.

To combine the individual bandwidth-limited data cubes $A^{\mathrm{k}}$, we need to combine at each position $(x, y)$ the individual phase spectra $\varphi^{\mathrm{k}}(x, y, \omega)$ obtained with the different DFG settings. A key for achieving this task is the sample drift correction during the recording of each bandwidth-limited data cube. As outlined above, the sample drift correction ensures that the position uncertainty is less than the spatial resolution, thus ensuring that spectra of the same position $(x, y)$ are combined.

The remaining challenges and solutions for combining the individual spectra are shown in Fig. 2. We recorded sampledrift-corrected data cubes of the same sample area using the three different laser outputs shown in Fig. $2 b$ (numerated $k=$ I, II, III). In Fig. 2a (bottom) we show the bandwidth-limited nano-FTIR phase spectra $\varphi^{\mathrm{k}}(x, y, \omega)$ of six subsequent pixels on the polymer blend sample. For better comparison, and to demonstrate the reproducibility of the individual spectra, the upper panel of Fig. 2a displays all spectra plotted on top of each other. Within the overlapping spectral regions (marked by grey areas) we clearly observe the same spectral features in the adjacent bandwidthlimited nano-FTIR phase spectra. However, the spectra can be significantly offset against each other by up to 12 degrees, although all spectra are normalized to a reference spectrum (see above). We explain this phase offset $\Delta \varphi$ (marked in Fig. 2a) by a small unavoidable drift of the interferometer paths ( $L_{\mathrm{R}}$ and $L_{\mathrm{t}}$ in Fig. 1a), which occurs between the acquisition of the individual nano-FTIR spectra and the acquisition of the reference spectrum (see Supplementary Note 5 and Supplementary Fig. 3). A drift as small as $100 \mathrm{~nm}$ of $L_{\mathrm{R}}$ relative to $L_{\mathrm{t}}$ shifts the normalized nano-FTIR phase spectrum by about $\Delta \varphi=6$ degree, which is in the same order of magnitude as the 
phase shift produced by absorption in the sample. To reduce offset fluctuations below 1 degree, the path lengths $L_{\mathrm{R}}$ and $L_{\mathrm{t}}$ (of about $6 \mathrm{~cm}$ ) need to be stabilized with a precision better than $20 \mathrm{~nm}$, which, however, will require sophisticated technology development in the future. Here we tackled the problem by correcting the phase offset. To that end, we shift at each pixel $(x, y)$ the phase spectra $\varphi^{\mathrm{I}}(\omega)$ and $\varphi^{I I I}(\omega)$ by the constant phase values $c^{\mathrm{I}}=\overline{\varphi^{\mathrm{I}}(\omega)}-\overline{\varphi^{\mathrm{II}}(\omega)}$ and $c^{\mathrm{III}}=\overline{\varphi^{\mathrm{III}}(\omega)}-\overline{\varphi^{\mathrm{II}}(\omega)}$ (the average values are evaluated in the corresponding spectral overlap regions marked grey in Fig. 2), to match $\varphi^{\mathrm{II}}(\omega)$. The offset-corrected phase spectra are shown in Fig. 2c. We find that the spectral features (marked by dashed black circles) in the overlapping regions are now well matched for all pixels, thus verifying the validity and reliability of our rather simple offset-correction procedure. Finally, we combine the three offset-corrected spectra at each pixel to obtain a single-broadband nano-FTIR phase spectrum. To achieve a smooth transition between the spectral ranges, we multiply the phase spectra $\varphi^{\mathrm{k}}$ by the functions $F^{\mathrm{k}}(\omega)$ shown by the red, green and blue graphs in Fig. 2d. Subsequently, the spectra are summed up. The final broadband nano-FTIR phase spectra are shown in Fig. 2e. Altogether, this methodology enables an automatized combination of bandwidth-limited data cubes to one hyperspectral data cube.

Analogue to far-field FTIR spectroscopic imaging, we finally apply a baseline correction ${ }^{41,42}$ to each broadband (composite) nano-FTIR phase spectrum $\varphi(\omega)$. We select two frequencies $\omega_{\mathrm{b} 1}$ and $\omega_{\mathrm{b} 2}$ (marked in Fig. $2 \mathrm{f}$, where we know or can assume that the sample absorption is negligible, see Supplementary Fig. 5) and subtract the linear baseline defined by the phase values $\varphi\left(\omega_{\mathrm{b} 1}\right)$ and $\varphi\left(\omega_{\mathrm{b} 2}\right)$. A comparison of baseline-corrected spectra (Fig. 2f) with the corresponding uncorrected spectra (Fig. 2e, upper panel) clearly shows the reduction of fluctuations between neighbouring spectra. Hence, the baseline correction significantly improves the SNR in the images extracted from the hyperspectral data cube (see Supplementary Note 6 and Supplementary Fig. 4).

The recording of a bandwidth-limited data cube $A^{\mathrm{k}}$ consisting of 5,084 nano-FTIR spectra (that is, $82 \times 62$ pixels, Fig. 1d) took $(5,084 \times 1.66 \mathrm{~s}) / 3,600=2.3 \mathrm{~h}$ while the accumulated additional time needed for the sample repositioning was of less than $10 \mathrm{~min}$ (for more details see Supplementary Note 3). Thus, the recording of the hyperspectral data cube $A$ shown in Fig. 1d required $7.4 \mathrm{~h}$.

Hyperspectral chemical nanoimaging of a polymer blend. In a first application example, we demonstrate hyperspectral IR nanoimaging with a three-component polymer blend, showing that this novel tool can meet the strong demand for highly sensitive nanoscale chemical mapping of the spatial distribution and local chemical interaction of the components. The model system studied in this work is based on a fluorine copolymer (FP), an acrylic copolymer (AC), and a polystyrene latex (PS; for details and fabrication see 'Methods' section). Figure 3a shows the topography image of the about $170 \mathrm{~nm}$ thick spin-coated polymer blend on silicon, while the hyperspectral infrared data of this sample area are displayed in Fig. 1d. The good reproducibility of the individual spectra (see Fig. $3 \mathrm{~b}$ showing four sets of neighbouring point spectra at the sample positions A to D marked in Fig. 3a,d) allow for valuable multivariate analysis based on established procedures known from far-field IR spectroscopy, as we demonstrate in the following.

We first applied inter-spectral distance mapping, where we calculate for each pixel spectrum $\mathrm{S}(x, y)$ the multivariate spectral distance $\mathrm{D}(x, y)$ between the point spectrum and a reference spectrum (see 'Methods' section). The distance values are converted into colour scales and plotted as specifically coloured pixels at the positions $(x, y)$. In the resulting distance maps, high colour intensity denotes a small distance (high similarity) with the reference spectrum (and vice versa). With nano-FTIR spectra obtained from reference samples made of pure AC and FP components (black spectra in Fig. 3b) we obtained the distance (similarity) maps for the AC (red) and FP (blue) components shown in Fig. 3c. Superposition of the two maps (see 'Methods' section) yields a compositional map (Fig. 3d), which highlights areas with the highest relative content of each polymer. We observe homogenous but distinct red and blue areas for the two different references, indicating that the $\mathrm{AC}$ and FP components are not fully mixed but rather separated. On the red areas, representative spectra (B) match well with the AC reference (black reference spectrum in Fig. 3b), indicating the presence of pure AC (illustrated by situation B in Fig. 3g). Within the blue areas, interestingly, representative spectra ( $D$ in Fig. 3b) show FP peaks at low frequencies $\left(\omega<1,500 \mathrm{~cm}^{-1}\right)$ but also the $\mathrm{C}=\mathrm{O}$ peak of $\mathrm{AC}$ at $1,740 \mathrm{~cm}^{-1}$. It can be shown (Supplementary Fig. 6) that the spectra at position D are a linear superposition of the pure FP and AC spectra, which lets us conclude that both FP and $\mathrm{AC}$ are present within the volume probed by the near field below the tip apex (represented in Fig. $3 g$ by the reddish elliptical area below the tip apex). We conclude that FP forms cluster-like nanostructures, while AC is spread all over the sample surface. For that reason, the near field below the tip apex probes both the FP cluster and AC layer below the FP cluster (illustrated by situation $\mathrm{D}$ in Fig. 3g). We explain this finding by the differences in viscosity, molecular weight and/or chain stiffness. On the other hand, the clearly visible spatial dispersion of $\mathrm{AC}$ and $\mathrm{FP}$-avoiding the formation of percolation networks-indicates that AC and FP are miscible even at the nanoscale. Note that any improvement of film formation or film homogenization by mixing or optimizing drying conditions was beyond the scope of this study.

In Fig. 3d we also observe black areas on the polymer blend, indicating that the similarity of the local spectra ( $A$ in Fig. $3 \mathrm{~b}$ ) is low compared with the FP and AC reference spectra. We explain these areas by the dominating presence of PS (illustrated by situation $\mathrm{A}$ in Fig. 3g). Note that we did not perform distance mapping with PS references, as the peaks in nano-FTIR spectra of pure PS reference sample are comparably weak.

Interestingly, Fig. 3d reveals purple areas (marked C in Fig. 3d), which indicate local spectral differences compared with both the $\mathrm{AC}$ (red) and FP (blue) regions. Indeed, representative spectra (marked $\mathrm{C}$ in Fig. 3b) of the purple areas cannot be reproduced by a linear superposition of AC and FP reference spectra (see Supplementary Fig. 6). Compared with the red AC spectrum (B), the $\mathrm{C}=\mathrm{O}$ peak at $1,740 \mathrm{~cm}^{-1}$ is reduced, indicating that the amount of AC in the probing volume of the tip is reduced. On the other hand, the peak at $1,155 \mathrm{~cm}^{-1}$ is significantly increased. We attribute this finding to the presence of FP and its chemical interaction with AC. Note that chemical interactions are known to cause peak shifts ${ }^{33}$ and increase of peak heights ${ }^{43}$. Specifically, we assume a spectral shift of the $\mathrm{CF}_{2}$ stretching vibration of FP from $1,195 \mathrm{~cm}^{-1}$ towards lower frequencies, most likely due to the formation of hydrogen bonding between the C-F bonds of FP and the acrylic polymer chains ${ }^{44,45}$. Further, the $\mathrm{C}-\mathrm{O}$ stretching of the ester bond of $\mathrm{AC}$ at $1,155 \mathrm{~cm}^{-1}$ may be enhanced due to the chemical interaction. The concerted action of both effects could thus explain the enhanced peak at $1,155 \mathrm{~cm}^{-1}$ that is found in the purple regions, which consequently indicate the areas where the AC and FP components are well mixed.

We note that similar compositional maps as the ones of Fig. $3 c$,d can be obtained with far-field infrared reference spectra 

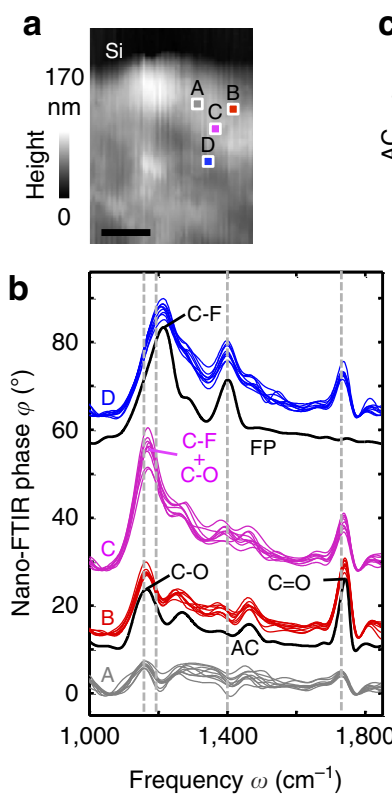

g
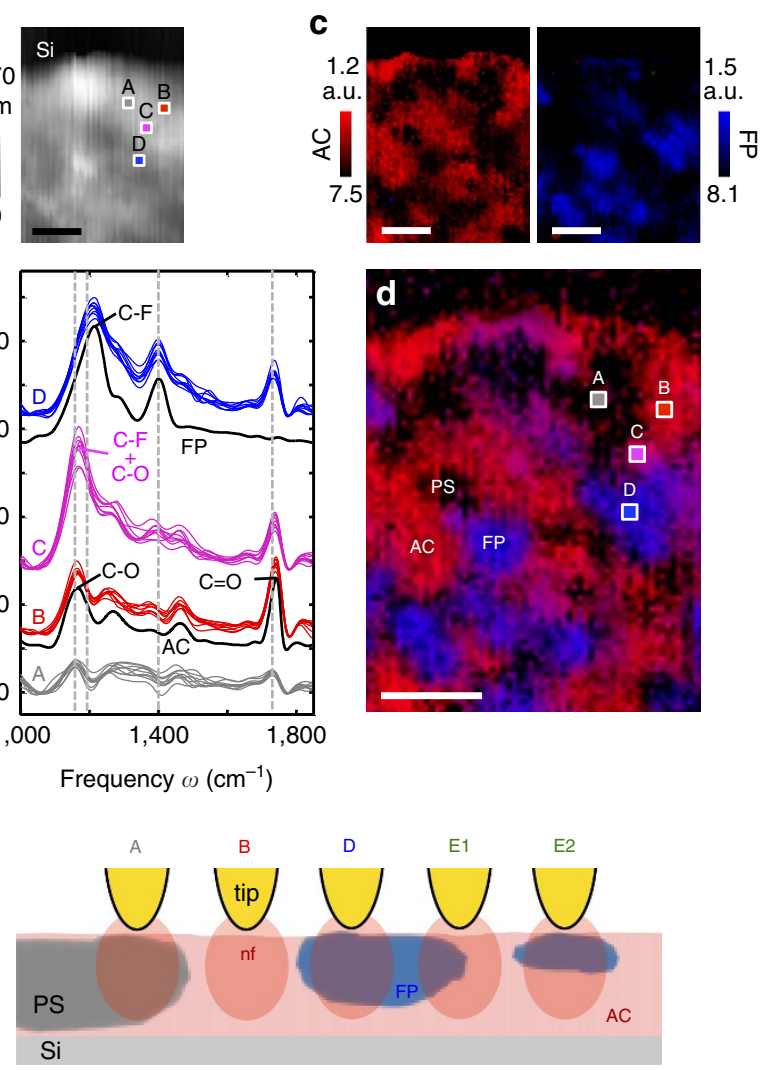
伿

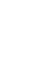


a
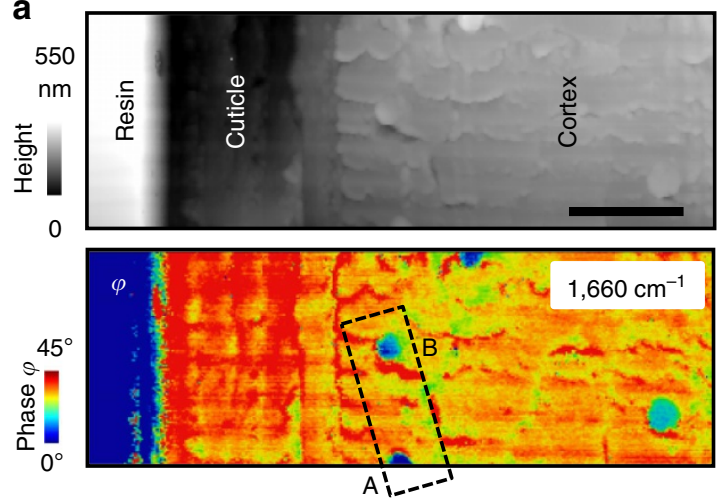

C

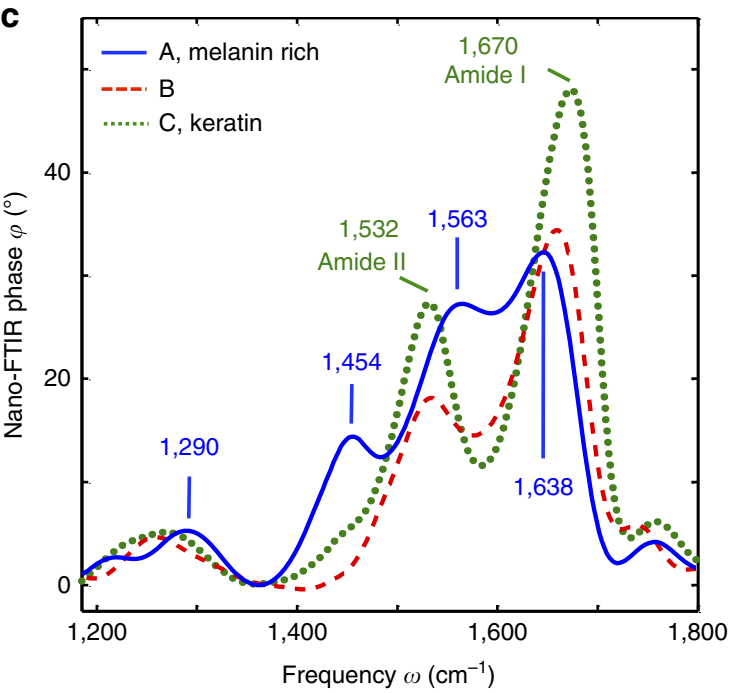

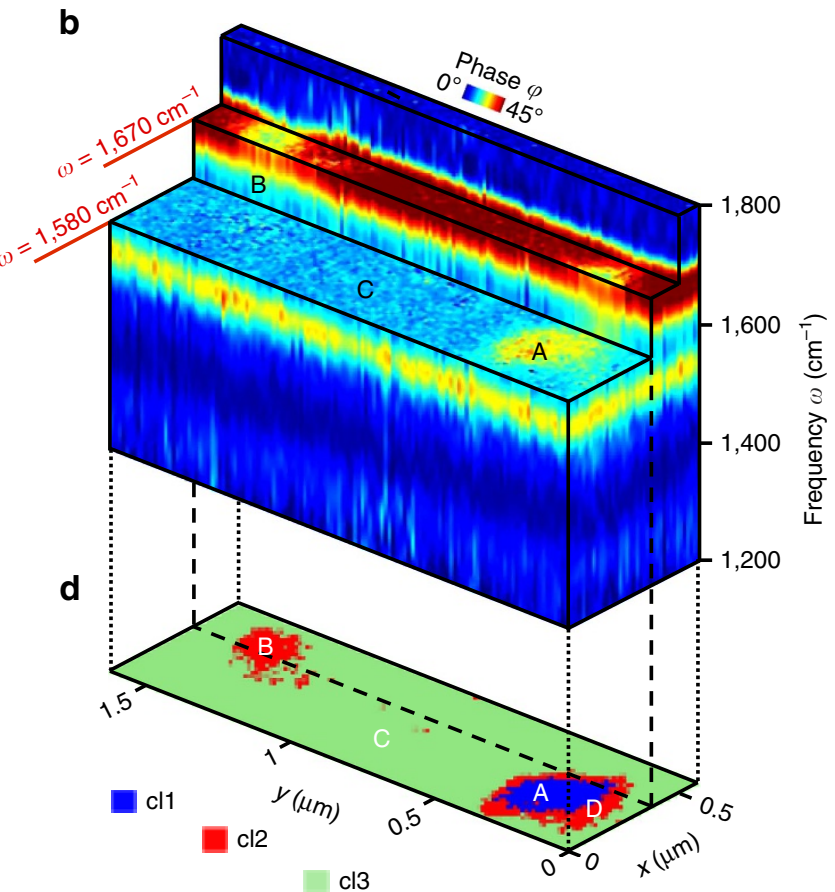

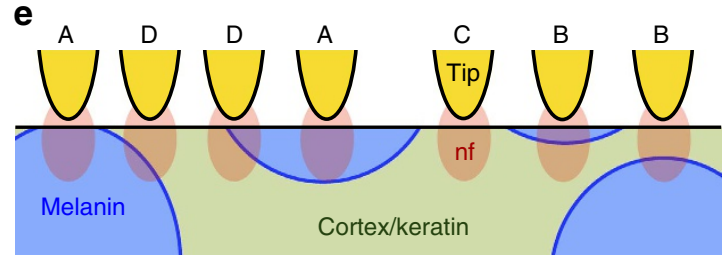

Figure 4 | In situ hyperspectral infrared nanoimaging of native melanin in human hair. (a) Top: Topography of a resin-embedded human hair microtome cross-section. Bottom: Infrared near-field phase image $\varphi$ at $1,660 \mathrm{~cm}^{-1}$. Scale bar, $1 \mu \mathrm{m}$. (b) Hyperspectral infrared data cube with a spectral resolution of $35 \mathrm{~cm}^{-1}$ partially cut at different frequencies $\omega$. It shows the phase of the tip-scattered light as a function of position $(x, y)$ and frequency $\omega$. The dashed black rectangle in a shows the area where the data cube was recorded. (c) Nano-FTIR spectra (average over nine neighbouring pixels) at positions marked by A, B and C in $\mathbf{a}, \mathbf{b}$ and $\mathbf{d}$, extracted from the data cube shown in $\mathbf{b}$. (d) Cluster map obtained by multivariate data analysis (HCA algorithm). (e) Schematics of near-field probing at positions A-D marked in $\mathbf{d}$.

extracting it from hair or tissue, which comes along with potential damage and modification ${ }^{51,52}$.

Figure $4 \mathrm{a}$ shows the topography of a resin-embedded cross-section of a hair. In the infrared near-field image taken with a quantum cascade laser at $1,660 \mathrm{~cm}^{-1}$ we observe an enhanced infrared absorption of the cuticle and cortex regions compared with the resin, owing to the strong amide I absorption of the hair proteins ( $\alpha$-keratin microfibrils). Within the cortex region we find disk-shaped areas of about $300 \mathrm{~nm}$ diameter, where the infrared absorption is reduced (that is, the protein content is reduced). Their size and distribution corresponds to that of melanin granules observed in electron microscopy images ${ }^{53}$. However, we found that nano-FTIR spectra of the individual granules can differ significantly from each other. To elucidate the spectroscopic variations, we performed hyperspectral IR nanoimaging of the area marked by dashed black line in Fig. 4a. From the hyperspectral data cube (Fig. 4b) we extracted spectra at different positions (Fig. 4c). Within the cortex region (position $\mathrm{C}$, green spectrum in Fig. 4c) we observe the well-known amide I and II bands being typical for protein $(\alpha$-keratin). For particle A (blue curve in Fig. 4c) we observe four distinct peaks that are characteristic for melanin: at $1,290 \mathrm{~cm}^{-1}$
(-C-OH phenolic stretching), $1,454 \mathrm{~cm}^{-1}$ (C-C aliphatic stretches), $1,563 \mathrm{~cm}^{-1}$ (indole $\mathrm{N}-\mathrm{H}$ bending) and $1,638 \mathrm{~cm}^{-1}$ $(\mathrm{C}=\mathrm{C}, \mathrm{C}=\mathrm{O} \text {, and/or COO- stretching in aromatic cycle })^{54}$. On particle $\mathrm{B}$-supposed to be a melanin granule-the nano-FTIR spectrum significantly differs from that of particle A. Surprisingly, spectrum B shows more similarity to the keratin spectrum of the cortex region (C), although the amid I and II peaks are shifted by several $\mathrm{cm}^{-1}$. Further, while both particles A and B are seen in the monochromatic image at $1,670 \mathrm{~cm}^{-1}$, only particle A exhibits a contrast at $1,580 \mathrm{~cm}^{-1}$ (see corresponding slices of the data cube in Fig. 4b). Obviously, a set of images and local spectra is not sufficient to clarify the identity of particle $B$. However, having acquired a full hyperspectral data cube, we can take advantage of multivariate data analysis. Since there are no reference spectra available for natural melanin in hair, we performed unsupervised hierarchical cluster analysis of the hyperspectral data. The segmentation map resulting from cluster analysis with three clusters (Fig. 4d) reveals well-defined features, thus corroborating the applicability and robustness of cluster analysis to the nano-FTIR spectra. We note that cluster analysis with more than three clusters does not reveal well-defined new features (see Supplementary Fig. 8). In both 
maps the particles $\mathrm{A}$ and $\mathrm{B}$ appear as homogenous, although as distinct clusters (circular blue and red areas, respectively). Most important, we find a red ring (D) around the blue central area of particle $\mathrm{A}$, revealing that the corresponding spectra belong to the same cluster as those of particle B. It can be shown that the spectra of the red cluster (B and D) are a linear superposition of spectra A and C (see Supplementary Fig. 9). For the width of the red ring (D) we measure about $50 \mathrm{~nm}$, which is in the range of the lateral spatial resolution. We thus conclude that the red ring highlights a steep interface between melanin and keratin (see illustrations D in Fig. 4e). The red area B, in contrast, is a closed disk-shaped area of about $200 \mathrm{~nm}$ diameter (that is, larger than the spatial resolution). We thus conclude that spectra $B$ of this area are due to a vertical arrangement of melanin and keratin, that is, a horizontally oriented interface between them. Indeed, near-field probing can be sensitive to subsurface components ${ }^{55}$, which lets us conclude that particle B is either a subsurface melanin granule or a thin slice of a melanin granule (see illustrations B in Fig. 4e; note that melanin granules are elliptical vesicles of an aspect ratio of about $3: 1$ ).

\section{Discussion}

The results presented in Figs 3 and 4 clearly demonstrate that hyperspectral data cubes of several 1,000 infrared spectra can be reliably obtained by nano-FTIR spectroscopy using a bandwidth-limited laser continuum. The hyperspectral data allow for multivariate analysis, providing nanoscale maps of the spatial distribution of organic materials. Most important, multivariate analysis reveals two types of spectral peak shifts within the individual nano-FTIR spectra of the polymer and hair samples. In the simplest case, individual nano-FTIR spectra exhibit peak shifts, which can be reproduced by a linear superposition of the nano-FTIR spectra of the pure reference samples (for example, on position D (blue area) on the polymer blend of Fig. 3, or on particle B in the hair sample of Fig. 4). It can be concluded that the spectra originate from the presence of the pure components (identical to that of the reference samples) in the volume probed by the near field below the tip. This can be the case, for example, at a sharp interface between pure components. In the second case, individual nano-FTIR spectra exhibit anomalous peak shifts, which cannot be reproduced by a linear superposition of the spectra of the pure reference samples. We conclude that the spectra originate from the presence of components, which are modified compared with the reference samples. We explain the modified spectral response by a chemical interaction between the adjacent components. Anomalous peak shifts have been found on the purple areas in Fig. 3e, which most likely occurred due to chemical interaction between the FP and AC components of the polymer blend.

Our studies of the hair cross-section of Fig. 4 demonstrate the capacity of hyperspectral infrared nanoimaging to identify and chemically analyse melanin molecules within their granula, without requiring deleterious extraction or tagging processes.
Through the analysis of the melanin-specific IR peak position and intensity in the future one could estimate the impact of various cosmetic treatments (like bleaching, ironing or colouring) on the chemical structure of the melanin polymer. Furthermore, analysing the protein secondary structure ${ }^{23}$ could allow for nanoscale spatial mapping of the impact of cosmetic treatments on the keratin fibrils and thus on the hair mechanical resistance.

In summary, we introduced techniques and methods for recording nanoscale-resolved hyperspectral infrared images obtained with a tunable bandwidth-limited mid-infrared laser continuum. It relies on recording multiple bandwidth-limited data cubes of one and the same sample area, and stitching them together to one hyperspectral data cube. Key for the reliable and accurate stitching of the data cubes has been the implementation of methods for (i) regular sample drift correction during data acquisition, (ii) regular recording of reference spectra during data acquisition, (iii) correcting spectral offsets caused by unavoidable interferometer drift and (iv) baseline correction of the broadband nano-FTIR spectra analogous to far-field hyperspectral infrared imaging. Using a tunable DFG laser continuum with an effective spectral bandwidth of about $350 \mathrm{~cm}^{-1}$, hyperspectral data cubes of about 5,000 nano-FTIR spectra covering $1,000-1,900 \mathrm{~cm}^{-1}$ could be recorded in less than 8 hours. Imaging a three-component polymer blend, we demonstrated that standard multivariate data analysis can be applied to the data, which allows for the automatized generation of chemical maps revealing material clustering and chemical interaction. We also studied a cross section of human hair, demonstrating the possibility of in situ multivariate nanoscale infrared analysis of individual melanin granules.

We foresee a wide application potential of hyperspectral infrared nanoimaging in various fields of science and technology, ranging from materials sciences and pharmaceutical applications to biomedical imaging, and from fundamental research to quality control. In combination with multivariate data analysis, hyperspectral infrared nanoimaging yields nanoscale-resolved chemical and compositional maps, including the detection of nanoscale localized chemical interaction. By improving the power and spectral bandwidth of emerging infrared laser sources (a recent work demonstrates a $100 \mathrm{~cm}^{-1}$ broad laser continuum of about $100 \mathrm{~mW}$ that is tunable between $500 \mathrm{~cm}^{-1}$ and $3,000 \mathrm{~cm}^{-1}$ ref. 56) and taking advantage of advanced spectral noise reduction strategies (that is, principal component analysis based noise reduction), we envision high-quality, hyperspectral infrared nanoimaging with few wavenumber spectral resolution on a time scale of one hour.

\section{Methods}

Sample preparation. Three water-based polymer dispersions made by emulsion polymerization were employed in this work. Table 1 summarizes their main characteristics.

Three-component polymer blend for hyperspectral infrared nanoimaging: The three polymer dispersions listed in Table 1 were separately diluted at $0.2 \%$ solids content and mixed into a single dispersion by stirring. Then, the mixture was casted onto a silicon wafer through spin coating. Finally, the silicon wafers were dried in an oven overnight at $120^{\circ} \mathrm{C}$. Proportions were chosen in

Table 1 | Main characteristics of the three water-based polymer dispersions employed to prepare the polymer blend.

Composition (\% weight)

Particle diameter, dp (nm)

PVDF/HFP

MMA/BA/AA

$P S / A A$
$95 / 5$

$49.5 / 49.5 / 1$

$99 / 1$
129

144

109

AA, acrylic acid; BA, butyl acrylate; HFP, hexafluoropropylene; MMA, methyl methacrylate; PS, polystyrene latex; PVDF, polyvinyledene fluoride. 
order to obtain a blend for coating applications. The final dispersion contained $40 \%$ of a polyvinyledene fluoride (PVDF) and hexafluoropropylene (HFP) copolymer; $40 \%$ of an acrylic copolymer made of methyl methacrylate (MMA), butyl acrylate (BA) and acrylic acid (AA); and $20 \%$ of a high glass transition temperature $\left(T_{\mathrm{g}}\right)$ latex based on polystyrene to improve mechanical properties without the need of any film forming aids ${ }^{57}$.

Polymer reference samples for nano-FTIR spectroscopy: The three polymer dispersions listed in Table 1 were separately diluted at $0.2 \%$ solids content. Each one was individually casted onto a silicon wafer through spin coating. Finally, the silicon wafers were dried in an oven overnight at $120^{\circ} \mathrm{C}$.

Polymer reference samples for ATR-FTIR spectroscopy: The three polymer dispersions listed in Table 1 were diluted at $1 \%$ and casted onto separate glass substrates and dried overnight at $120^{\circ} \mathrm{C}$. The obtained polymer layers were several $\mu \mathrm{m}$ thick.

Hair sample preparation. A clean human hair was cut into small pieces and embedded in epoxy resin (Epon kit substitute embedding medium, Aldrich). The sample was cured during $24 \mathrm{~h}$ at $60^{\circ} \mathrm{C}$. The block containing the human hair was trimmed with fresh glass knife made with Leica knifemaker and then ultramicrotomed with Leica UCT using Diatome $45^{\circ}$ diamond knife.

FTIR spectroscopy. ATR-FTIR of the hair cross section. We used a Bruker Hyperion 2,000 microscope coupled to a Vertex 70 FTIR spectrometer equipped with an ATR module ( $\times 20$ ATR objective, Bruker, single-internal reflection) comprising a germanium crystal with a diameter of about $100 \mu \mathrm{m}$ at the point of contact with the sample. The spectrum (showing the absorbance) was measured with a spectral resolution of $4 \mathrm{~cm}^{-1}$ and presents an average over 250 scans with a total acquisition time of $215 \mathrm{~s}$.

ATR-FTIR of polymers. We used a Frontier FTIR/FIR spectrometer (Perkin Elmer) equipped with a universal ATR module (1 reflection with diamond/ ZnSe top plates). The spectra were measured with a spectral resolution of $4 \mathrm{~cm}^{-1}$ and present an average over 150 scans with a total acquisition time of $796 \mathrm{~s}$. The reference spectra were recorded on air. All ATR-FTIR spectra show the absorbance.

Multivariate analysis of hyperspectral infrared nanoimages. Inter-spectral distance mapping (Fig. 3) was carried out by means of the CytoSpec software package (CytoSpec, Berlin, Germany). Distance images were constructed by calculating Euclidean distances between the external reference spectra and the preprocessed nano-FTIR spectra contained in the hyperspectral data sets. In this approach, distance values were converted to colour scales and plotted as a function of the spatial $(x, y)$ coordinates. In distance imaging, a high colour intensity denotes a low inter-spectral distance, or high similarity, between the actual point spectrum and a given reference spectrum. The CytoSpec software package also allows for constructing so-called composite images that are superpositions of distinct distance maps.

CytoSpec was also used for image reassembling based on cluster analysis (Figs 3 and 4). Basic principles of this image segmentation technique have been published elsewhere ${ }^{5,58}$. In this study, $D$-values were used as inter-spectral distance measures and Ward's method was employed as the clustering method. To calculate the multivariate distance values, the spectral range from 1,000 to $1,850 \mathrm{~cm}^{-1}$ was utilized.

Data availability. The data that support the findings of this study are available from the corresponding author upon reasonable request.

\section{References}

1. Salzer, R. \& Siesler, H. W. Infrared and Raman Spectroscopic Imaging: Second, Completely Revised and Updated Edition (Wiley, 2014).

2. Coleman, M. M. \& Painter, P. C. Fourier transform infrared spectroscopy: probing the structure of multicomponent polymer blends. Appl. Spectrosc. Rev. 20, 255-346 (1984)

3. Diem, M., Romeo, M., Boydston-White, S., Miljković, M. \& Matthäus, C. A decade of vibrational micro-spectroscopy of human cells and tissue (1994-2004). Analyst 129, 880-885 (2004).

4. Levin, I. W. \& Bhargava, R. Fourier transform infrared vibrational spectroscopic imaging: integrating microscopy and molecular recognition. Annu. Rev. Phys. Chem. 56, 429-474 (2005).

5. Baker, M. J. et al. Using Fourier transform IR spectroscopy to analyze biological materials. Nat. Protoc. 9, 1771-1791 (2014).

6. Dazzi, A., Prazeres, R., Glotin, F. \& Ortega, J. M. Local infrared microspectroscopy with subwavelength spatial resolution with an atomic force microscope tip used as a photothermal sensor. Opt. Lett. 30, 2388-2390 (2005).

7. Nowak, D. et al. Nanoscale chemical imaging by photoinduced force microscopy. Sci. Adv. 2, e1501571-e1501571 (2016)

8. Lu, F., Jin, M. \& Belkin, M. A. Tip-enhanced infrared nanospectroscopy via molecular expansion force detection. Nat. Photon. 8, 307-312 (2014).
9. Knoll, B. \& Keilmann, F. Near-field probing of vibrational absorption for chemical microscopy. Nature 399, 134-137 (1999).

10. Keilmann, F. \& Hillenbrand, R. Nano-Optics and Near-Field Optical Microscopy (Artech House, 2008).

11. Amarie, S. \& Keilmann, F. Broadband-infrared assessment of phonon resonance in scattering-type near-field microscopy. Phys. Rev. B 83, 45404 (2011).

12. Huth, F., Schnell, M., Wittborn, J., Ocelic, N. \& Hillenbrand, R. Infraredspectroscopic nanoimaging with a thermal source. Nat. Mater. 10, 352-356 (2011).

13. Amarie, S. et al. Nano-FTIR chemical mapping of minerals in biological materials. Beilstein J. Nanotechnol. 3, 312-323 (2012).

14. Huth, F. et al. Nano-FTIR absorption spectroscopy of molecular fingerprints at $20 \mathrm{~nm}$ spatial resolution. Nano Lett. 12, 3973-3978 (2012).

15. Xu, X. G., Rang, M., Craig, I. M. \& Raschke, M. B. Pushing the sample-size limit of infrared vibrational nanospectroscopy: from monolayer toward single molecule sensitivity. J. Phys. Chem. Lett. 3, 1836-1841 (2012).

16. Govyadinov, A. A., Amenabar, I., Huth, F., Carney, P. S. \& Hillenbrand, R. Quantitative measurement of local infrared absorption and dielectric function with tip-enhanced near-field microscopy. J. Phys. Chem. Lett. 4, 1526-1531 (2013).

17. Hermann, P. et al. Near-field imaging and nano-Fourier-transform infrared spectroscopy using broadband synchrotron radiation. Opt. Express 21, 2913-2919 (2013).

18. Bechtel, H. A., Muller, E. A., Olmon, R. L., Martin, M. C. \& Raschke, M. B. Ultrabroadband infrared nanospectroscopic imaging. Proc. Natl Acad. Sci. USA 111, 7191-7196 (2014).

19. Peragut, F., Brubach, J.-B., Roy, P. \& De Wilde, Y. Infrared near-field imaging and spectroscopy based on thermal or synchrotron radiation. Appl. Phys. Lett. 104, 251118 (2014).

20. O'Callahan, B. T. et al. Broadband infrared vibrational nano-spectroscopy using thermal blackbody radiation. Opt. Express 23, 32063-32074 (2015).

21. Jones, A. C. \& Raschke, M. B. Thermal infrared near-field optical spectroscopy. Nano Lett. 12, 1475-1481 (2012).

22. Keilmann, F. \& Amarie, S. Mid-infrared frequency comb spanning an octave based on an Er fiber laser and difference-frequency generation. J. Infrared Millim. Terahertz Waves 33, 479-484 (2012).

23. Amenabar, I. et al. Structural analysis and mapping of individual protein complexes by infrared nanospectroscopy. Nat. Commun. 4, 2890 (2013).

24. Xu, X. G. \& Raschke, M. B. Near-field infrared vibrational dynamics and tip-enhanced decoherence. Nano Lett. 13, 1588-1595 (2013).

25. Khatib, O. et al. Graphene-based platform for infrared near-field nanospectroscopy of water and biological materials in an aqueous environment ACS Nano 9, 7968-7975 (2015).

26. Hermann, P. et al. Characterization of semiconductor materials using synchrotron radiation-based near-field infrared microscopy and nano-FTIR spectroscopy. Opt. Express 22, 17948-17958 (2014).

27. Mastel, S., Govyadinov, A. A., de Oliveira, T. V. A. G., Amenabar, I. \& Hillenbrand, R. Nanoscale-resolved chemical identification of thin organic films using infrared near-field spectroscopy and standard Fourier transform infrared references. Appl. Phys. Lett. 106, 23113 (2015).

28. Hillenbrand, R., Taubner, T. \& Keilmann, F. Phonon-enhanced light-matter interaction at the nanometre scale. Nature 418, 159-162 (2002).

29. Hoffmann, J. M., Hauer, B. \& Taubner, T. Antenna-enhanced infrared near-field nanospectroscopy of a polymer. Appl. Phys. Lett. 101, 193105 (2012).

30. Kehr, S. C. et al. Anisotropy contrast in phonon-enhanced apertureless near-field microscopy using a free-electron laser. Phys. Rev. Lett. 100, 256403 (2008).

31. Stiegler, J. M. et al. Nanoscale free-carrier profiling of individual semiconductor nanowires by infrared near-field nanoscopy. Nano Lett. 10, 1387-1392 (2010).

32. Taubner, T., Hillenbrand, R. \& Keilmann, F. Nanoscale polymer recognition by spectral signature in scattering infrared near-field microscopy. Appl. Phys. Lett. 85, 5064-5066 (2004).

33. Pollard, B., Muller, E. A., Hinrichs, K. \& Raschke, M. B. Vibrational nanospectroscopic imaging correlating structure with intermolecular coupling and dynamics. Nat. Commun. 5, 3587 (2014).

34. Brehm, M., Taubner, T., Hillenbrand, R. \& Keilmann, F. Infrared spectroscopic mapping of single nanoparticles and viruses at nanoscale resolution. Nano Lett 6, 1307-1310 (2006)

35. Muller, E. A., Pollard, B., Bechtel, H. A., van Blerkom, P. \& Raschke, M. B. Infrared vibrational nano-crystallography and nano-imaging. Sci. Adv. 2, e1601006 (2016).

36. Wueppen, J., Jungbluth, B., Taubner, T. \& Loosen, P. in Infrared, Millimeter and Terahertz Waves (IRMMW-THz), 36th-International Conference, IEEE, $1-2(2011)$ 
37. Bensmann, S. et al. Near-field imaging and spectroscopy of locally strained GaN using an IR broadband laser. Opt. Express 22, 22369-22381 (2014).

38. Labardi, M., Patane, S. \& Allegrini, M. Artifact-free near-field optical imaging by apertureless microscopy. Appl. Phys. Lett. 77, 621-623 (2000).

39. Hillenbrand, R. \& Keilmann, F. Complex optical constants on a subwavelength scale. Phys. Rev. Lett. 85, 3029-3032 (2000).

40. Salmons, B. S., Katz, D. R. \& Trawick, M. L. Correction of distortion due to thermal drift in scanning probe microscopy. Ultramicroscopy 110, 339-349 (2010).

41. Mazet, V., Carteret, C., Brie, D., Idier, J. \& Humbert, B. Background removal from spectra by designing and minimising a non-quadratic cost function. Chemom. Intell. Lab. Syst. 76, 121-133 (2005).

42. Lasch, P. Spectral pre-processing for biomedical vibrational spectroscopy and microspectroscopic imaging. Chemom. Intell. Lab. Syst. 117, 100-114 (2012).

43. Coleman, M. M. \& Painter, P. C. Hydrogen bonded polymer blends. Prog. Polym. Sci. 20, 1-59 (1995).

44. Leonard, C., Halary, J. L. \& Monnerie, L. Crystallization of poly (vinylidene fluoride)-poly (methyl methacrylate) blends: analysis of the molecular parameters controlling the nature of poly (vinylidene fluoride) crystalline phase. Macromolecules 21, 2988-2994 (1988).

45. Mohamadi, S. in Infrared Spectroscopy-Materials Science, Engineering and Technology (ed. Theophanides, T.) 213-232 (InTech, Chapters published, 2012).

46. Sturgeon, R. J. A re-investigation of the borohydride reduction of carbohydrates. Carbohydr. Res. 227, 375-377 (1992).

47. Milligan, G. W. \& Cooper, M. C. An examination of procedures for determining the number of clusters in a data set. Psychometrika 50, 159-179 (1985).

48. Brenner, M. \& Hearing, V. J. The protective role of melanin against UV damage in human skin. Photochem. Photobiol. 84, 539-549 (2008).

49. Marcott, C. et al. Localization of human hair structural lipids using nanoscale infrared spectroscopy and imaging. Appl. Spectrosc. 68, 564-569 (2014).

50. Ihssen, J., Braun, A., Faccio, G., Gajda-Schrantz, K. \& Thöny-Meyer, L. Light harvesting proteins for solar fuel generation in bioengineered photoelectrochemical cells. Curr. Protein Pept. Sci. 15, 374 (2014).

51. Liu, Y. et al. Comparison of the structural and physical properties of human hair eumelanin following enzymatic or acid/base extraction. Pigment Cell Res. 16, 355-365 (2003)

52. Liu, Y. et al. Comparison of structural and chemical properties of black and red human hair melanosomes. Photochem. Photobiol. 81, 135-144 (2005).

53. Sato, S., Sasaki, Y., Adachi, A. \& Omi, T. Reduction and block staining of human hair shafts and insect cuticles by ammonium thioglycolate to enhance transmission electron microscopic observations. J. Cosmet. Dermatological Sci. Appl. 3, 157-161 (2013).

54. Bridelli, M. G. \& Crippa, P. R. Infrared and water sorption studies of the hydration structure and mechanism in natural and synthetic melanin. J. Phys. Chem. B 114, 9381-9390 (2010).

55. Taubner, T., Keilmann, F. \& Hillenbrand, R. Nanoscale-resolved subsurface imaging by scattering-type near-field optical microscopy. Opt. Express 13, 8893-8899 (2005).

56. Steinle, T., Mörz, F., Steinmann, A. \& Giessen, H. Ultra-stable high average power femtosecond laser system tunable from 1 . 33 to $20 \mu \mathrm{m}$. Opt. Lett. 41, 4863-4866 (2016).
57. Feng, J., Winnik, M. A., Shivers, R. R. \& Clubb, B. Polymer blend latex films: morphology and transparency. Macromolecules 28, 7671-7682 (1995).

58. Lasch, P., Haensch, W., Naumann, D. \& Diem, M. Imaging of colorectal adenocarcinoma using FT-IR microspectroscopy and cluster analysis. Biochim. Biophys. Acta 1688, 176-186 (2004).

\section{Acknowledgements}

We thank the Chemical Engineering group of the University of Basque CountryPOLYMAT for providing the polymer dispersions, Dr Gracia Patricia Leal for preparing microtome cuts of the hair, and Dr Michael Laue (RKI Berlin) for fruitful discussions and support. We acknowledge support from the European Union through the ERC starting grant TERATOMO (grant no. 258461), the Spanish Ministry of Economy and Competitiveness (national projects MAT-2015-65525-R) and the Department of Industry of the Basque Government (ETORTEK 2014-CORROPTO project)

\section{Author contributions}

I.A. and R.H. conceived the study. I.A. implemented hyperspectral imaging, and performed nano-FTIR imaging experiments and data analysis. M.G. and W.N performed far-field FTIR spectroscopy. S.P. contributed to the scientific interpretation of the hair spectra. M.G prepared the polymer sample and contributed to the scientific interpretation of the polymer spectra. P.L. provided multispectral data analysis software and contributed to data analysis. R.H. coordinated and supervised the study. I.A. and R.H wrote the manuscript with input from all authors. All authors contributed to the scientific discussion and manuscript revisions.

\section{Additional information}

Supplementary Information accompanies this paper at http://www.nature.com/ naturecommunications

Competing financial interests: R.H. is co-founder of Neaspec GmbH, a company producing scattering-type scanning near-field optical microscope systems such as the one used in this study. P.L. is the author and owner of CytoSpec, a software package for vibrational hyperspectral imaging which was used in this study. The remaining authors declare no competing financial interests.

Reprints and permission information is available online at http://npg.nature.com/ reprintsandpermissions/

How to cite this article: Amenabar, I. et al. Hyperspectral infrared nanoimaging of organic samples based on Fourier transform infrared nanospectroscopy. Nat. Commun. 8, 14402 doi: $10.1038 /$ ncomms14402 (2017).

Publisher's note: Springer Nature remains neutral with regard to jurisdictional claims in published maps and institutional affiliations.

This work is licensed under a Creative Commons Attribution 4.0 International License. The images or other third party material in this article are included in the article's Creative Commons license, unless indicated otherwise in the credit line; if the material is not included under the Creative Commons license, users will need to obtain permission from the license holder to reproduce the material. To view a copy of this license, visit http://creativecommons.org/licenses/by/4.0/

(C) The Author(s) 2017 